1 Hacettepe Journal of Mathematics and Statistics

$\bigcap$ Volume 47 (6) (2018), 1512-1520

\title{
Closure, interior and neighbourhood in a category
}

\author{
David Holgate $^{* \dagger}$ and Josef Šlapal ${ }^{\ddagger}$
}

\begin{abstract}
The natural correspondences in topology between closure, interior and neighbourhood no longer hold in an abstract categorical setting where subobject lattices are not necessarily Boolean algebras. We analyse three canonical correspondences between closure, interior and neighbourhood operators in a category endowed with a subobject structure. While these correspondences coincide in general topology, the analysis highlights subtle differences which distinguish different approaches taken in the literature.
\end{abstract}

Dedicated to the memory of Professor Lawrence M. Brown.

Keywords: Categorical closure operator, interior operator, neighbourhood operator.

Mathematics Subject Classification (2010): 18A20, 18B30, 54A05, 54A20, 54B30, $54 \mathrm{C} 10$

Received : 19.09.2016 Accepted : 30.11.2016 Doi : 10.15672/HJMS.2018.628

\section{Introduction}

The articles [9] and [10] spawned a programme of research into closure operators in categories. Using the notion of closure operator - and later simply axiomatically treated families of "closed" morphisms [7] - topological properties were studied in general categories. Purely categorical proofs were given for topological theorems with, perhaps surprisingly, little recourse to the idempotence and/or additivity of the closure in question. The books [11] and [2] provide surveys of this programme while [7] and references therein give immediate access to the central articles.

Until more recently little had been done to consider the "dual" notion to closure, namely interior, as primitive. For some time, the only article to explicitly consider

*Department of Mathematics and Applied Mathematics, University of the Western Cape, Bellville 7535, South Africa, Email: dholgate@uwc.ac.za

${ }^{\dagger}$ Corresponding Author.

‡IT4Innovations Centre of Excellence, Brno University of Technology, Božetěchova 2, 61266 Brno, Czech Republic, Email: slapal@fme.vutbr.cz 
interior operators for a number of years was [20]. This has since been followed by [3, 4, 16], amongst others. While closure and interior may be "dual" at an intuitive level they only characterise each other when the subobject lattices on which they operate are Boolean algebras. (Or at least have an appropriate involution as noted in [20].)

Openness with respect to a closure operator is considered in [14]. There it is also observed that a smooth transition between open and closed is only possible if the subobject lattices have appropriate complements. What is clearly evident in that article is that morphisms which are continuous with respect to a closure operator are by no means "continuous" with respect to the associated open subobjects. The fact that closure and interior operators are required to act functorially, and not simply in the subobject lattices, is what precludes an easy "duality" between the two.

More recently, in [12], [13] and [18], neighbourhoods with respect to a categorical closure operator were introduced and used to study topological properties. The principal motivation being that neighbourhoods, as against closure operators, provide the appropriate tool for introducing convergence in a category (a categorical approach to convergence was discussed in [17]). Again their transition from closure to neighbourhood is made via (pseudo)complements and many results rely on the existence of (pseudo)complements for their validity.

In [15] and [19] neighbourhood operators are introduced as primitive and used to then study compactness in particular. It is this introduction of a general neighbourhood operator that motivated the current note to analyse more systematically how closure, interior and neighbourhood operators interact, and to provide a framework within which a number of existing investigations can be understood.

We observe that there is a one-one correspondence between neighbourhood and interior operators and present three correspondences between interior (equivalently neighbourhood) and closure operators.

\section{Preliminaries}

Categorical terminology follows [1], while for closure operators we refer to [11] or [2]. We consider a category $\mathcal{C}$ and a fixed class $\mathcal{M}$ of $\mathcal{C}$-monomorphisms. $\mathcal{C}$ is $\mathcal{M}$-complete, i.e. pullbacks of $\mathcal{M}$-morphisms along arbitrary $\mathcal{C}$-morphisms and arbitrary intersections of $\mathcal{M}$-morphisms exist and are again in $\mathcal{M}$.

As a consequence, there is an $(\mathbb{E}, \mathcal{M})$ factorisation system for sinks (or co-cones) in $\mathcal{C}$. (Cf. [1] Chapter 15 for the dual results.) When $(\mathbb{E}, \mathcal{M})$ is restricted to morphisms in $\mathcal{C}$, we denote the resulting factorisation system by $(\mathcal{E}, \mathcal{M})$. This affords each $f: X \rightarrow Y$ a unique factorisation $f=m \cdot e$ with $e \in \mathcal{E}$ and $m \in \mathcal{M}$.

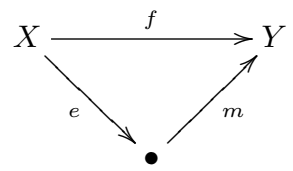

For a given $X \in \mathcal{C}, \operatorname{sub} X:=\{m \in \mathcal{M} \mid$ codomain of $m$ is $X\}$. These "subobjects" are ordered by $n \leq m \Leftrightarrow m \cdot j=n$ for some $j$ (necessarily in $\mathcal{M}$ ). If $n \leq m$ and $m \leq n$ then they are isomorphic. We do not distinguish between isomorphic subobjects, treating $\operatorname{sub} X$ as a lattice and writing $m=n$ for isomorphic subobjects.

Our assumptions on $\mathcal{M}$ render $\operatorname{sub} X$ to be a complete lattice for each $X \in \mathcal{C}$. The least element of $\operatorname{sub} X$ is denoted by $0_{X}$ and the greatest by $1_{X}$ (which is the identity arrow on $X$ ).

A subobject $m^{*} \in \operatorname{sub} X$ is the pseudocomplement of $m \in \operatorname{sub} X$ if it holds that $n \leq m^{*} \Leftrightarrow n \wedge m=0_{X}$. If $m^{*}$ exists, $m$ is said to be pseudocomplemented. 
A complement of $m \in \operatorname{sub} X$ is an $\bar{m} \in \operatorname{sub} X$ such that $m \wedge \bar{m}=0_{X}$ and $m \vee \bar{m}=1_{X}$. If such an $\bar{m}$ exists then $m$ is said to be complemented in $\operatorname{sub} X$.

Any morphism $f: X \rightarrow Y$ in $\mathcal{C}$ induces an image/pre-image adjunction

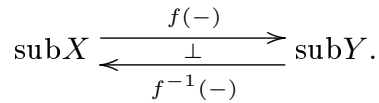

For $m \in \operatorname{sub} X, f(m)$ is the $\mathcal{M}$-component of the $(\mathcal{E}, \mathcal{M})$ factorisation of $f \cdot m$, while for $n \in \operatorname{sub} Y, f^{-1}(n)$ is the pullback of $n$ along $f$.

2.1. Definition. A morphism $f: X \rightarrow Y$ reflects 0 if $f^{-1}\left(0_{Y}\right)=0_{X}$ (equivalently $\left.f(m)=0_{Y} \Leftrightarrow m=0_{X}\right)$.

We will on occasion find it useful to impose that $\mathcal{E}$ is stable under pullback along $\mathcal{M}$-morphisms. This is well known to be equivalent to the so-called Frobenius reciprocity law holding, namely that given any $f: X \rightarrow Y$ and $m \in \operatorname{sub} X, y \in \operatorname{sub} Y$,

$$
f\left(m \wedge f^{-1}(n)\right)=f(m) \wedge n \text {. }
$$

2.2. Proposition. Let $\mathcal{E}$ be stable under pullback along $\mathcal{M}$-morphisms. A morphism $f: X \rightarrow Y$ reflects 0 if and only if $f^{-1}(-)$ preserves pseudocomplements.

Proof. Let $f$ reflect 0 and let $n \in \operatorname{sub} Y$ have pseudocomplement $n^{*}$. Observe that $m \leq$ $f^{-1}\left(n^{*}\right) \Leftrightarrow f(m) \leq n^{*} \Leftrightarrow f(m) \wedge n=0_{Y} \Leftrightarrow f\left(m \wedge f^{-1}(n)\right)=0_{Y} \Leftrightarrow m \wedge f^{-1}(n)=0_{X}$, so $f^{-1}\left(n^{*}\right)=f^{-1}(n)^{*}$.

On the other hand if $f^{-1}(-)$ preserves pseudocomplements then $f^{-1}\left(0_{Y}\right)=f^{-1}\left(1_{Y}^{*}\right)=$ $f^{-1}\left(1_{Y}\right)^{*}=1_{X}^{*}=0_{X}$.

Note that in particular if the subobject lattices are Boolean algebras then under the conditions of the above proposition $f^{-1}(-)$ preserves complements.

We are interested in the interplay of three types of operators acting on the subobject lattices.

2.3. Definition. A closure operator $c$ on $\mathcal{C}$ with respect to $\mathcal{M}$ is a family

$$
\left\{c_{X}: \operatorname{sub} X \rightarrow \operatorname{sub} X \mid X \in \mathcal{C}\right\}
$$

of functions such that

(C1) $m \leq c_{X}(m)$ for every $m \in \operatorname{sub} X$ and $X \in \mathcal{C}$,

(C2) $m \leq n \Rightarrow c_{X}(m) \leq c_{X}(n)$ for every $m, n \in \operatorname{sub} X$ and $X \in \mathcal{C}$,

(C3) every morphism $f: X \rightarrow Y$ in $\mathcal{C}$ is c-continuous, $f\left(c_{X}(m)\right) \leq c_{Y}(f(m))$ for every $m \in \operatorname{sub} X$.

We extend the subobject ordering pointwise to closure operators, writing $c \leq c^{\prime}$ if $c(m) \leq c^{\prime}(m)$ for all $m \in \mathcal{M}$. (We will sometimes drop the subscript of $c_{X}$ if clarity does not demand it.) The resulting ordered conglomerate of all closure operators on $\mathcal{C}$ with respect to $\mathcal{M}$ will be denoted $\mathrm{CL}(\mathcal{C}, \mathcal{M})$.

2.4. Definition. An interior operator $i$ on $\mathcal{C}$ with respect to $\mathcal{M}$ is a family

$$
\left\{i_{X}: \operatorname{sub} X \rightarrow \operatorname{sub} X \mid X \in \mathcal{C}\right\}
$$

of functions such that

(I1) $i_{X}(m) \leq m$ for every $m \in \operatorname{sub} X$ and $X \in \mathcal{C}$,

(I2) $m \leq n \Rightarrow i_{X}(m) \leq i_{X}(n)$ for every $m, n \in \operatorname{sub} X$ and $X \in \mathcal{C}$,

(I3) every morphism $f: X \rightarrow Y$ in $\mathcal{C}$ is $i$-continuous, $f^{-1}\left(i_{Y}(m)\right) \leq i_{X}\left(f^{-1}(m)\right)$ for every $m \in \operatorname{sub} Y$. 
As for closure, the subobject ordering is extended pointwise to interior operators, the ordered conglomerate of all interior operators on $\mathcal{C}$ with respect to $\mathcal{M}$ being denoted $\operatorname{INT}(\mathcal{C}, \mathcal{M})$.

2.5. Definition. A neighbourhood operator $\nu$ on $\mathcal{C}$ with respect to $\mathcal{M}$ is a family

$$
\left\{\nu_{X}: \operatorname{sub} X \rightarrow \mathcal{P}(\operatorname{sub} X) \mid X \in \mathcal{C}\right\}
$$

of functions such that $\nu_{X}(m)$ is a stack for each $m$ and

(N1) $n \in \nu_{X}(m) \Rightarrow m \leq n$ for every $m \in \operatorname{sub} X$ and $X \in \mathcal{C}$,

(N2) $m \leq n \Rightarrow \nu_{X}(m) \supseteq \nu_{X}(n)$ for every $m, n \in \operatorname{sub} X$ and $X \in \mathcal{C}$,

(N3) If $\mathcal{G} \subseteq \operatorname{sub} X$ and $n \in \nu_{X}(m)$ for all $m \in \mathcal{G}$ then $n \in \nu_{X}(\bigvee \mathcal{G})$,

(N4) every morphism $f: X \rightarrow Y$ in $\mathcal{C}$ is $\nu$-continuous, $f^{-1}(\nu(m)) \subseteq \nu\left(f^{-1}(m)\right)$ for every $m \in \operatorname{sub} Y$.

Note that we introduce the convention $f^{-1}(\nu(m))=\left\{f^{-1}(n) \mid n \in \nu(m)\right\}$. The fact that we work with neighbourhoods of subspaces allows (N4) to be given in clear analogy to (C3) and (I3). In point set topology when neighbourhoods of points are considered, such an axiom remains hidden.

Neighbourhood operators are again ordered pointwise, $\nu \leq \nu^{\prime}$ if $\nu(m) \subseteq \nu^{\prime}(m)$ for all $m \in \mathcal{M}$. The ordered conglomerate of all neighbourhood operators on $\mathcal{C}$ with respect to $\mathcal{M}$ is denoted $\mathrm{NBH}(\mathcal{C}, \mathcal{M})$.

For the rest of this section we will use the symbols $c$, $i$ and $\nu$ to denote closure, interior and neighbourhood operators respectively, without explicitly stating so.

Note that the image/preimage adjunction (2.1) allows continuity of $f: X \rightarrow Y$ (in the presence of the other axioms) to be equivalently described for the respective operators as follows:

$\left(\mathrm{C} 3^{\prime}\right) c_{X}\left(f^{-1}(n)\right) \leq f^{-1}\left(c_{Y}(n)\right)$ for every $n \in \operatorname{sub} Y$,

$\left(\mathrm{I} 3^{\prime}\right) f(m) \leq i_{Y}(n) \Rightarrow m \leq i_{X}\left(f^{-1}(n)\right)$ for every $n \in \operatorname{sub} Y$,

$\left(\mathrm{N}^{\prime}\right) n \in \nu_{Y}(f(m)) \Rightarrow f^{-1}(n) \in \nu_{X}(m)$ for every $m \in \operatorname{sub} X$,

$\left(\mathrm{N}^{\prime \prime}\right) n \in \nu_{Y}(f(m)) \Rightarrow \exists p \in \nu_{X}(m)$ with $f(p) \leq n$ for every $m \in \operatorname{sub} X$.

2.6. Definition. A closure/interior/neighbourhood operator is grounded if

(C0) $c_{X}\left(0_{X}\right)=0_{X}$ for all $X \in \mathcal{C}$,

(I0) $i_{X}\left(1_{X}\right)=1_{X}$ for all $X \in \mathcal{C}$,

(N0) $1_{X} \in \nu_{X}(m)$ for all $X \in \mathcal{C}$ and $m \in \operatorname{sub} X$.

2.7. Definition. These operators have naturally associated notions of closed or open subobjects. A subobject $m \in \operatorname{sub} X$ is termed

- c-closed if $m=c_{X}(m)$,

- $i$-open if $m=i_{X}(m)$,

- $\nu$-open if $m \in \nu_{X}(m)$, (i.e. if $\nu_{X}(m)=\uparrow m$, the principal filter in subX generated by $m$ ).

The operator is idempotent if for every $m \in \mathcal{M}$,

- $c(m)$ is $c$-closed,

- $i(m)$ is $i$-open,

- $\bigvee\{n \mid m \in \nu(n)\}$ is $\nu$-open.

2.8. Definition. Finally we term the operator additive if for any $X \in \mathcal{C}$ and $m, n \in$ $\operatorname{sub} X$ :

- $c_{X}(m \vee n)=c_{X}(m) \vee c_{X}(n)$,

- $i_{X}(m \wedge n)=i_{X}(m) \wedge i_{X}(n)$,

- $\nu_{X}(m)$ is a filter. 


\section{Interior and neighbourhood}

3.1. Definition. Let $M \stackrel{m}{\rightarrow} X \in \mathcal{M}$. For an interior operator $i$, put

$$
\nu_{X}^{i}(m):=\left\{n \in \operatorname{sub} X \mid m \leq i_{X}(n)\right\}
$$

and for a neighbourhood operator $\nu$, put

$$
i_{X}^{\nu}(m):=\bigvee\left\{n \in \operatorname{sub} X \mid m \in \nu_{X}(n)\right\} .
$$

3.2. Proposition. For an interior operator $i$ and neighbourhood operator $\nu$ on $\mathcal{C}$ with respect to $\mathcal{M}$, the assignments

$$
i \mapsto \nu^{i} \text { and } \nu \mapsto i^{\nu}
$$

define an order preserving isomorphism between $\operatorname{InT}(\mathcal{C}, \mathcal{M})$ and $\mathrm{NBH}(\mathcal{C}, \mathcal{M})$.

Proof. First we note that for any $M \stackrel{m}{\rightarrow} X \in \mathcal{M}, \nu_{X}^{i}(m)$ is clearly a stack, and furthermore:

(N1) $m \leq i_{X}(n) \leq n$ for all $n \in \nu_{X}^{i}(m)$.

(N2) If $m \leq n$, then $n \leq i_{X}(p) \Rightarrow m \leq i_{X}(p)$ and $\nu_{X}^{i}(n) \subseteq \nu_{X}^{i}(m)$.

(N3) $\mathcal{G} \subseteq \operatorname{sub} X, m \leq i_{X}(n)$ for all $m \in \mathcal{G} \Rightarrow \bigvee \mathcal{G} \leq i_{X}(n) \Rightarrow n \in \nu_{X}^{i}(\bigvee \mathcal{G})$

(N4) Let $f: X \rightarrow Y$ and $n \in \nu_{Y}^{i}(m)$. Then $m \leq i_{Y}(n) \Rightarrow f^{-1}(m) \leq f^{-1}\left(i_{Y}(n)\right) \leq$ $i_{X}\left(f^{-1}(n)\right)$, so $f^{-1}(n) \in \nu_{X}^{i}\left(f^{-1}(m)\right)$.

On the other hand given $M \stackrel{m}{\rightarrow} X \in \mathcal{M}$, we see that:

(I1) $m \in \nu_{X}(n) \Rightarrow n \leq m \Rightarrow i_{X}^{\nu}(m) \leq m$.

(I2) If $m \leq n$ then $m \in \nu_{X}(p) \Rightarrow n \in \nu_{X}(p)$ (since $\nu_{X}(p)$ is a stack) and hence $i_{X}^{\nu}(m) \leq i_{X}^{\nu}(n)$.

(I3) Take $f: X \rightarrow Y$ and $n \in \operatorname{sub} Y$. By (N3), $n \in \nu_{Y}\left(i_{Y}^{\nu}(n)\right)$. Then from (N4) we have, $f^{-1}(n) \in \nu_{X}\left(f^{-1}\left(i_{Y}^{\nu}(n)\right)\right)$. So by the definition of $i^{\nu}, f^{-1}\left(i_{Y}^{\nu}(n)\right) \leq$ $i_{X}^{\nu}\left(f^{-1}(n)\right)$.

It is clear that the assignments $i \mapsto \nu^{i}$ and $\nu \mapsto i^{\nu}$ preserve order. To see that they are mutually inverse we note that for $M \stackrel{m}{\rightarrow} X \in \mathcal{M}$ :

(1) $i_{X}^{\nu^{i}}(m)=\bigvee\left\{n \in \operatorname{sub} X \mid m \in \nu_{X}^{i}(n)\right\}=\bigvee\left\{n \in \operatorname{sub} X \mid n \leq i_{X}(m)\right\}=i_{X}(m)$.

(2) $n \in \nu_{X}^{i^{\nu}}(m) \Leftrightarrow m \leq i_{X}^{\nu}(n) \Rightarrow \nu_{X}\left(i_{X}^{\nu}(n)\right) \subseteq \nu_{X}(m)$. Thus since (as observed above) $n \in \nu_{X}\left(i_{X}^{\nu}(n)\right), n \in \nu_{X}(m)$. On the other hand, $n \in \nu_{X}(m) \Rightarrow m \leq$ $i_{X}^{\nu}(n) \Rightarrow n \in \nu_{X}^{i^{\nu}}(m)$.

3.3. Remarks. A few immediate observations about the above equivalence.

(1) Evidently grounded operators coincide since a $\nu_{X}(m)$ is non-empty if and only if $1_{X} \in \nu_{X}(m)$. It is also easy to see that $i$ is additive precisely when $\nu^{i}$ is too.

(2) It is clear from the definition of $i^{\nu}$ that $m=i^{\nu}(m) \Leftrightarrow m \in \nu(m)$. Restating the definition of idempotence for neighbourhood operators, we have that $\nu$ is idempotent if and only if $i^{\nu}(m) \in \nu\left(i^{\nu}(m)\right)$, that is if $i^{\nu}(m)=i^{\nu}\left(i^{\nu}(m)\right)$ for every $m \in \mathcal{M}$.

(3) Because of (N2) and (N3) we have that for any $m \in \operatorname{sub} X$,

$$
\begin{aligned}
\nu_{X}\left(i_{X}^{\nu}(m)\right) & =\nu_{X}\left(\bigvee\left\{n \in \operatorname{sub} X \mid m \in \nu_{X}(n)\right\}\right) \\
& =\bigcap\left\{\nu_{X}(n) \mid m \in \nu_{X}(n)\right\} .
\end{aligned}
$$

(4) Note that if $\nu$ is idempotent then $\bigcap\left\{\nu_{X}(n) \mid m \in \nu_{X}(n)\right\}$ is a principal filter for every $m \in \mathcal{M}$. This property does not characterise idempotence, however. 


\section{From interior to closure}

There are a number of candidates for defining closed subobjects relative to a given interior or neighbourhood operator. All base their intuition on the associated notions in topology and thus coincide when the subobject lattices are Boolean algebras. We begin by considering three possibilities.

4.1. Definition. Let $i \in \operatorname{Int}(\mathcal{C}, \mathcal{M}), X \in \mathcal{C}$ and $m \in \operatorname{sub} X$. We say that $m$ is:

(1) $A^{i}$-closed if $i_{X}(m \vee n) \leq m \vee i_{X}(n)$ for all $n \in \operatorname{sub} X$.

(2) $B^{i}$-closed if $m \vee n=1_{X} \Rightarrow m \vee i_{X}(n)=1_{X}$ for all $n \in \operatorname{sub} X$.

(3) $C^{i}$-closed if $m$ is pseudocomplemented and $m^{*}=i_{X}\left(m^{*}\right)$.

4.2. Proposition. Let $i \in \operatorname{InT}(\mathcal{C}, \mathcal{M})$ and $M \stackrel{m}{\rightarrow} X \in \mathcal{M}$.

(1) If $i$ is grounded, then $m$ is $A^{i}$-closed $\Rightarrow m$ is $B^{i}$-closed.

(2) If subX is a Boolean algebra, $m$ is $B^{i}$-closed $\Leftrightarrow m$ is $C^{i}$-closed.

(3) If $i$ is additive and subX is a Boolean algebra, then $m$ is $C^{i}$-closed $\Rightarrow m$ is $A^{i}$-closed.

Proof. (1) If $i$ is grounded then $1_{X}=m \vee n=i_{X}(m \vee n) \leq m \vee i_{X}(n) \Rightarrow m \vee i_{X}(n)=1_{X}$.

(2) If $\operatorname{sub} X$ is a Boolean algebra, $m^{*}=\bar{m}$. If $m$ is $B^{i}$-closed then $m \vee \bar{m}=1_{X} \Rightarrow$ $m \vee i_{X}(\bar{m})=1_{X} \Rightarrow i_{X}(\bar{m}) \geq \bar{m} \Rightarrow \bar{m}=i_{X}(\bar{m})$. Conversely if $\bar{m}=i_{X}(\bar{m})$ then $m \vee n=1_{X} \Rightarrow \bar{m} \leq n \Rightarrow \bar{m}=i_{X}(\bar{m}) \leq i_{X}(n) \Rightarrow m \vee i_{X}(n)=1_{X}$.

(3) Assume that $i$ is additive, $\operatorname{sub} X$ is a Boolean algebra and $m^{*}=i_{X}\left(m^{*}\right)$. Since we are in a Boolean algebra, $m^{*}=\bar{m}$. For any $n \in \operatorname{sub} X$ :

$$
\begin{aligned}
i_{X}(m \vee n) \wedge \bar{m} & =i_{X}(m \vee n) \wedge i_{X}(\bar{m}) \\
& =i_{X}((m \vee n) \wedge \bar{m}) \\
& =i_{X}(n \wedge \bar{m}) \\
& =i_{X}(n) \wedge i_{X}(\bar{m}) \\
& =i_{X}(n) \wedge \bar{m}
\end{aligned}
$$

Thus for any $n \in \operatorname{sub} X$ we conclude:

$$
\begin{aligned}
i_{X}(m \vee n) & =i_{X}(m \vee n) \wedge(m \vee \bar{m}) \\
& =\left(i_{X}(m \vee n) \wedge m\right) \vee\left(i_{X}(m \vee n) \wedge \bar{m}\right) \\
& =m \vee\left(i_{X}(n) \wedge \bar{m}\right) \\
& =\left(m \vee i_{X}(n)\right) \wedge(m \vee \bar{m}) \\
& =m \vee i_{X}(n)
\end{aligned}
$$

4.3. A standard technique for generating a closure operator from a class $\mathcal{F} \subseteq \mathcal{M}$ is as follows. (Cf. [5] or [6] for a proof.)

(1) Form the "stabilisation" $\mathcal{F}^{*}=\left\{f^{-1}(m) \mid m \in \mathcal{F}, \operatorname{cod}(m)=\operatorname{cod}(f)\right\}$ of $\mathcal{F}$. Clearly $\mathcal{F} \subseteq \mathcal{F}^{*} \subseteq \mathcal{M}$

(2) For $M \stackrel{m}{\rightarrow} X \in \mathcal{M}$ the assignment

$$
c_{X}(m)=\bigwedge\left\{n \in \mathcal{F}^{*} \mid m \leq n\right\}
$$

defines an idempotent closure operator $c \in \mathrm{CL}(\mathcal{X}, \mathcal{M})$.

It is easy to verify that every $m \in \mathcal{F}$ is $c$-closed and that $c$ is the largest closure operator on $\mathcal{C}$ with respect to $\mathcal{M}$ for which this is the case.

4.4. Definition. Let $i \in \operatorname{InT}(\mathcal{C}, \mathcal{M})$. Denote by $\alpha^{i}, \beta^{i}$ and $\gamma^{i}$ the closure operators obtained by performing the construction in (4.1) for $\mathcal{F}$ the class of $A^{i}$-closed, $B^{i}$-closed and $C^{i}$-closed $\mathcal{M}$-morphisms, respectively. 
The assignments $i \mapsto \beta^{i}$ and $i \mapsto \gamma^{i}$ define order reversing maps from $\operatorname{InT}(\mathcal{C}, \mathcal{M})$ to $\mathrm{CL}_{\mathrm{L}}(\mathcal{C}, \mathcal{M})$. The assignment $i \mapsto \alpha^{i}$ does not respect order in general. (Assuming $\mathcal{C}_{-}$ morphisms reflect 0 , the interior operators $i_{1}(m)=0$ and $i_{2}(m)=m$ for all $m \in \mathcal{M}$ both induce the closure operator $\alpha^{i}(m)=m$ for all $m \in \mathcal{M}$.)

Of course, if the class $\mathcal{F}$ is already pullback stable, then the construction in (4.1) simply amounts to $c_{X}(m)=\bigwedge\{n \mid n$ is closed and $m \leq n\}$ for an appropriate notion of "closed" (i.e. being in $\mathcal{F}$ ). The following proposition points out that this will be the case in a number of instances.

4.5. Proposition. Let $i \in \operatorname{InT}(\mathcal{C}, \mathcal{M})$. If $(\mathcal{E}, \mathcal{M})$ is stable and every $f \in$ More reflects 0 , then $C^{i}$-closed morphisms are closed under pullback.

Proof. Let $f: X \rightarrow Y$ and $m \in \operatorname{sub} Y$ be $C^{i}$-closed. Using Proposition 2.2 we have: $f^{-1}(m)^{*}=f^{-1}\left(m^{*}\right)=f^{-1}\left(i_{Y}\left(m^{*}\right)\right) \leq i_{X}\left(f^{-1}\left(m^{*}\right)\right)=i_{X}\left(f^{-1}(m)^{*}\right)$.

\section{From closure to interior}

The construction of an interior (or neighbourhood) operator analogous to the closure operator construction above is facilitated if the factorisation system for sinks, $(\mathbb{E}, \mathcal{M})$ is what we shall term $\mathcal{M}$-stable, namely that if $\left\{e_{\lambda}: A_{\lambda} \rightarrow Y\right\}_{\lambda \in I} \in \mathbb{E}$ with each $e_{\lambda} \in \mathcal{M}$ and $f: X \rightarrow Y$, then $\left\{f^{-1}\left(e_{\lambda}\right)\right\}_{\lambda \in I} \in \mathbb{E}$. This is equivalent to requiring that joins commute with pre-image, as is true for example of functions on sets,

$$
f^{-1}\left(\bigvee_{m \in \mathcal{S}} m\right)=\bigvee_{m \in \mathcal{S}} f^{-1}(m) \text { for any } \mathcal{G} \subseteq \operatorname{sub} X \text { and } X \in \mathcal{C} .
$$

A further equivalent way to state this condition is to say that for every $f: X \rightarrow Y$ in $\mathrm{C}$, the pre-image map $f^{-1}(-): \operatorname{sub} Y \rightarrow \operatorname{sub} X$ is both left and right adjoint. It is right adjoint to the image map $f(-)$ and left adjoint to the assignment described by $\tilde{f}(m)=\bigvee\left\{p \in \operatorname{sub} Y \mid f^{-1}(p) \leq m\right\}$.

Given a class $\mathcal{F} \subseteq \mathcal{M}$, perform the following construction. First form the stabilisation $\mathcal{F}^{*}$, then for $M \stackrel{m}{\rightarrow} X \in \mathcal{M}$ assign

$$
i_{X}(m)=\bigvee\left\{p \in \mathcal{F}^{*} \mid p \leq m\right\}
$$

5.1. Proposition. Let $(\mathbb{E}, \mathcal{M})$ be $\mathcal{M}$-stable and let $\mathcal{F} \subseteq \mathcal{M}$. The construction of $i(m)$ for $m \in \mathcal{M}$ given in (5.1) above defines an idempotent interior operator $i \in \operatorname{INT}(\mathcal{C}, \mathcal{M})$.

Proof. (I1), (I2) and idempotence are trivial. $\mathcal{M}$-stability of $(\mathbb{E}, \mathcal{M})$ and pullback stability of $\mathcal{F}^{*}$ ensure (I3).

5.2. Remarks. (1) Clearly every $m \in \mathcal{F}$ is $i$-open and $i$ is the smallest interior operator for which this is the case.

(2) One can of course avoid the assumption of $(\mathbb{E}, \mathcal{M})$ being $\mathcal{M}$-stable by iterating the stabilisation of $\mathcal{F}$ under pullback and join tansfinitely.

Analogously to Definition 4.1, Proposition 4.2 and Definition 4.4 we have the following development.

5.3. Definition. Let $c \in \mathrm{CL}_{\mathrm{L}}(\mathcal{C}, \mathcal{M}), X \in \mathcal{C}$ and $m \in \operatorname{sub} X$. We say that $m$ is:

(1) $\mathfrak{A}^{c}$-open if $m \wedge c_{X}(n) \leq c_{X}(m \wedge n)$ for all $n \in \operatorname{sub} X$.

(2) $\mathfrak{B}^{c}$-open if $m \wedge n=0_{X} \Rightarrow m \wedge c_{X}(n)=0_{X}$ for all $n \in \operatorname{sub} X$.

(3) $\mathfrak{C}^{c}$-open if $m$ is pseudocomplemented and $m^{*}=c_{X}\left(m^{*}\right)$.

5.4. Proposition. Let $c \in \mathrm{CL}(\mathcal{C}, \mathcal{M})$ and $M \stackrel{m}{\rightarrow} X \in \mathcal{M}$.

(1) If $c$ is grounded, then $m$ is $\mathfrak{A}^{c}$-open $\Rightarrow m$ is $\mathfrak{B}^{c}$-open. 
(2) If subX is a Boolean algebra then $m$ is $\mathfrak{B}^{c}$-open $\Rightarrow m$ is $\mathfrak{C}^{c}$-open.

(3) If $c$ is additive and subX is a Boolean algebra, then $m$ is $\mathfrak{C}^{c}$-open $\Rightarrow m$ is $\mathfrak{A}^{c}$ open.

Proof. Analogous to that of Proposition 4.2 except that in (2) we need that $m^{*}=\bar{m}$.

5.5. Definition. Let $(\mathbb{E}, \mathcal{M})$ be $\mathcal{M}$-stable and let $c \in C_{L}(\mathcal{C}, \mathcal{M})$. Denote by $\mathfrak{a}^{c}, \mathfrak{b}^{c}$ and $\mathfrak{c}^{c}$ the interior operators defined as in (5.1) for $\mathcal{F}$ the class of $\mathfrak{A}^{c}$-open, $\mathfrak{B}^{c}$-open and $\mathfrak{C}^{c}$-open $\mathcal{M}$-morphisms respectively.

As in Proposition 4.5 we can prove:

5.6. Proposition. If $(\mathcal{E}, \mathcal{M})$ is stable and morphisms in $\mathcal{C}$ reflect 0 , then for any closure operator $c$, the $\mathfrak{C}^{c}$-open morphisms are pullback stable.

5.7. We have established a number of correspondences. These are not in general Galois connections (one assignment does not even preserve order in general) but they do have a certain "duality" to them. When the subobject lattices in $\mathcal{C}$ are Boolean algebras then, paired alphabetically and considering grounded operators, they render Galois connections between $\operatorname{INT}(\mathcal{C}, \mathcal{M}) \cong \mathrm{NBH}(\mathcal{C}, \mathcal{M})$ and $\mathrm{CL}(\mathcal{C}, \mathcal{M})$.

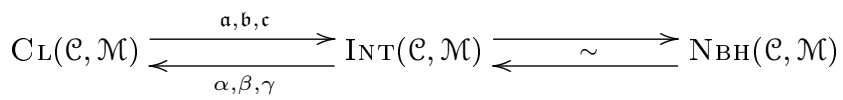

In this simplified setting, the various notions of closed and open collapse and $m$ is open $\Leftrightarrow \bar{m}$ is closed. This is essentially the analysis conducted in [20].

When the category $\mathcal{C}$ is a topological construct, then taking $\mathcal{M}$ to be the embeddings (initial injective maps) not only are the subobject lattices Boolean algebras but in addition $(\mathbb{E}, \mathcal{M})$ is $\mathcal{M}$-stable, $(\mathcal{E}, \mathcal{M})$ is stable and all morphisms reflect 0 . In these examples there will be small technical differences if the operators being considered are not grounded or additive, otherwise the three correspondences $\operatorname{INT}(\mathcal{C}, \mathcal{M}) \stackrel{\alpha, \beta, \gamma}{\rightarrow} \mathrm{CL}(\mathcal{C}, \mathcal{M})$ coincide as do $\mathrm{CL}(\mathcal{C}, \mathcal{M}) \stackrel{\mathfrak{a}, \mathfrak{b}, \mathfrak{c}}{\rightarrow} \operatorname{INT}(\mathcal{C}, \mathcal{M})$. Their composition can be used to describe the idempotent hull of a closure operator or (taken in the other direction) the idempotent core of an interior/neighbourhood operator.

Of the other articles where interior and/or neighbourhood operators related to a closure operator are studied, [12], [13] and [20] use our third notion, $\mathfrak{C}^{c}$-open, in the transition from closure to neighbourhood/interior. This third notion of openness and its counterpart for closedness, $C^{i}$-closed, is of little use however in typically algebraic settings where the subobject lattices are not (pseudo)complemented.

Our first notion of closed/open $\left(A^{i}\right.$-closed, $\mathfrak{A}^{c}$-open) is that considered in [14]. We are not aware of our second notion appearing in the closure operator literature. We propose this to be the most natural definition.

Regarding algebraic examples, the type of construction in (4.1) has recently received rejuvenated interest in work of Guiterres and Clementino [8]. In [15] a different construction of a closure operator from a neighbourhood operator is considered.

Acknowledgement. The first author acknowledges the support of the South African National Research Foundation. The second author acknowledges the support from the Ministry of Education, Youth and Sports of the Czech Republic under the National Programme of Sustainability (NPU II) project "IT4Innovations Excellence in Science LQ1602". 


\section{References}

[1] Adámek, J., Herrlich, H., Strecker, G.E. Abstract and concrete categories, Whiley, 1990.

[2] Castellini, G. Categorical closure operators, Mathematics: Theory \& Applications Birkhäuser Boston Inc., Boston, MA, 2003.

[3] Castellini, G. Interior operators in a category: idempotency and heredity, Topology Appl. 158 (17), 2332-2339, 2011.

[4] Castellini, G. Some remarks on interior operators and the functional property, Quaest. Math. 39 (2), 275-287, 2016.

[5] Castellini, G., Holgate, D. Closure operator constructions depending on one parameter, Quaest. Math. 26 (3), 289-305, 2003.

[6] Castellini, G., Koslowski, J., Strecker, G.E. An approach to the dual of regular closure operators, Cahiers Topologie Géom. Différentielle Catégoriques 35, 109-128, 1994.

[7] Clementino, M. M., Giuli, E., Tholen, W. A functional approach to general topology, in: Categorical foundations 97, Encyclopedia Math. Appl., 103-163, Cambridge Univ. Press, Cambridge, 2004.

[8] Clementino, M. M., Gutierres, G. On regular and homological closure operators, Cah. Topol. Géom. Différ. Catég. 51 (2), 127-142, 2010.

[9] Dikranjan, D., Giuli, E. Closure operators I, Topology Appl. 27 (2), 129-143, 1987.

[10] Dikranjan, D., Giuli, E., Tholen, W. Closure operators II, in: Categorical topology and its relation to analysis, algebra and combinatorics, Prague, 1988, 297-335, World Sci. Publishing, Teaneck, NJ, 1989.

[11] Dikranjan, D., Tholen, W. Categorical structure of closure operators, Kluwer Academic Publishers Group, Dordrecht, 1995.

[12] Giuli, E., Šlapal, J. Raster convergence with respect to a closure operator, Cah. Topol. Géom. Différ. Catég. 46 (4), 275-300, 2005.

[13] Giuli, E., Šlapal, J. Neighborhoods with respect to a categorical closure operator, Acta Math. Hungar. 124 (1-2), 1-14, 2009.

[14] Giuli, E., Tholen, W. Openness with respect to a closure operator, Appl. Categ. Structures 8 (3), 487-502, 2000.

[15] Holgate, D., Šlapal, J. Categorical neighborhood operators, Topology Appl. 158 (17), 2356$2365,2011$.

[16] Razafindrakoto, A., Holgate, D. Interior and neighbourhood, Topology Appl. 168, 144-152, 2014.

[17] Šlapal, J., Net spaces in categorical topology, Ann. N. Y. Acad. Sci. 806, 393-412, 1996.

[18] Šlapal, J., Neighborhoods and convergence with respect to a closure operator, Math. Slovaca 61 (5), 717-732, 2011.

[19] Šlapal, J., Compactness and convergence with respect to a neighborhood operator, Collect. Math. 63 (2), 123-137, 2012.

[20] Vorster, S. J. R., Interior operators in general categories, Quaest. Math. 23 (4), 405-416, 2000 . 